\title{
The ExaVolt Antenna Mission Concept and Technology Developments
}

\author{
Andrew Romero-Wolf ${ }^{* a}$, Peter Gorham ${ }^{b}$, Patrick Allison ${ }^{c}$, Frank Baginski ${ }^{d}$, Jim \\ Beatty $^{c}$, Ken Brakke ${ }^{e}$, Amy Connolly ${ }^{c}$, Brian Dailey ${ }^{c}$, Debra Fairbrother ${ }^{f}$, Joshua \\ Furer $^{d}$, Brian Hill ${ }^{b}$, Eugene Hong ${ }^{c}$, Steven Lang ${ }^{f}$, Kurt Liewer $^{a}$, Christian Miki ${ }^{b}$, Liem \\ Nguyen $^{b}$, Carl Pfendner ${ }^{c}$, Magdi Said $f$, Harm Schoorlemmer ${ }^{b}$, Gary Varner $^{b}$, Leyland \\ Young ${ }^{f}$, Kaiyu Zhao ${ }^{d}$ \\ ${ }^{a}$ Jet Propulsion Laboratory, California Institute of Technology, Pasadena, CA 91109 USA \\ ${ }^{b}$ Department of Physics, University of Hawaii at Manoa, Honolulu, HI 96822 USA \\ ${ }^{c}$ Department of Physics, Ohio State University, Columbus, OH 43210 USA \\ ${ }^{d}$ Department of Mathematics, George Washington University, Washington, DC 20052 USA \\ e Department of Mathematical Sciences, Susquehanna University, Selinsgrove, PA 17870 USA \\ ${ }^{f}$ NASA Balloon Program Office, NASA Wallops Flight Facility, Wallops Island, VA 20337 USA \\ E-mail: Andrew.Romero-Wolfejpl.nasa.gov
}

\begin{abstract}
In the past decade, searches for the cosmogenic neutrino flux produced by the interactions of ultra-high energy cosmic rays with the cosmic microwave background have not yet resulted in detection. Radio detection of ultra-high energy neutrinos provides a cost-effective means probing large amounts of effective volume. The Antarctic Impulsive Transient Antenna (ANITA) balloonborne experiment, with sensitivity to neutrinos with energies $>10^{19} \mathrm{eV}$, has provided some of the most stringent limits on cosmogenic neutrino production models by searching for coherent radio Cherenkov signals produced by the Askaryan effect in Antarctic ice. The ExaVolt Antenna (EVA) is a mission concept to extend the sensitivity of balloon-borne radio neutrino detection to energies $10^{17} \mathrm{eV}$. EVA uses a novel antenna design that exploits the surface area of the balloon to provide a reflector antenna with $30 \mathrm{dBi}$ gain (compared to $10 \mathrm{dBi}$ on ANITA). We will present an overview of the mission concept and recent technology developments.
\end{abstract}

The 34th International Cosmic Ray Conference,

30 July- 6 August, 2015

The Hague, The Netherlands

\footnotetext{
* Speaker.
} 


\section{Introduction}

The ultra-high energy (UHE) cosmogenic neutrino flux was first predicted by Berezinsky and Zatsepin in 1969 [1] as the result of UHE cosmic ray interactions with the cosmic background radiation. This flux of extragalactic neutrinos in the Exavolt $\left(1 \mathrm{EeV}=10^{18} \mathrm{eV}\right)$ energy range $[2,3]$ must be present at a level that is constrained by the known existence, emerging composition, and unknown cosmic evolution of the sources of the ultra-high energy cosmic rays (UHECR) [4].

Several experiments are currently pursuing the detection of the cosmogenic UHE neutrino flux using radio detection techniques. Detectors embedded within polar ice caps several kilometers deep $[5,6]$ exploit the proximity to neutrino interactions in a large target volume. The surface detectors of ARIANNA [7] overlook the highly reflective ocean 600-m beneath the Ross Ice Shelf enabling sensitivity to downgoing neutrinos. The ANITA [8] balloon-borne interferometer, at an altitude of $\sim 37 \mathrm{~km}$, synoptically scans a significantly larger ice volume $\left(\sim\right.$ million $\left.\mathrm{km}^{3}\right)$ providing high sensitivity to the high energy end of the expected neutrino spectra. The sensitivities of these experiments and models of the UHE cosmogenic neutrino flux are shown in Figure 1.

The combination of visible target volume and live-time of the experiment determine the sensitivity to UHE-induced radio emissions. Ground-based arrays implement large numbers of high duty cycle stations (37 for ARA and 1000 for ARIANNA) where the visible volume is limited by the index of refraction gradient of the ice. A balloon-borne detector, on the other hand, has a large visible target volume with a limited operational duty cycle. For balloon-borne detectors, the only practical improvement for this technique is to increase the antenna gain. The ExaVolt antenna (EVA) [9] is a mission concept that builds on the success of three ANITA flights. EVA aims to implement a $\gtrsim 30 \mathrm{dBi}$ gain antenna, exploiting the surface of a super-pressure balloon (SPB) to im-

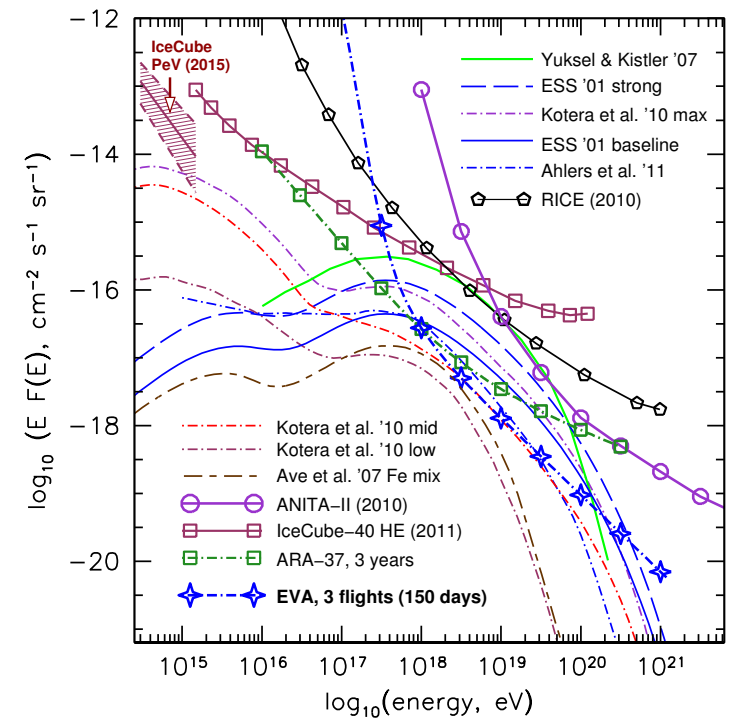

Figure 1: UHE neutrino models, experimental limits including published values from the 2008 ANITA-II flight, 3years of operation of the planned ARA-37 [10] embedded detector at the South Pole, and a 150 day simulated exposure of EVA.
Table 1: Expected numbers of events $N_{v}$ for published values of ANITA-II, 3 years of ARA-37, and 150 days of EVA with $80 \%$ analysis efficiency.

\begin{tabular}{cccc}
\hline \hline Model \& references $\quad N_{v}:$ & $\begin{array}{c}\text { ANITA-II } \\
(2008)\end{array}$ & $\begin{array}{c}\text { ARA } \\
3 \mathrm{yr}\end{array}$ & $\begin{array}{c}\text { EVA } \\
150 \mathrm{~d}\end{array}$ \\
\hline Baseline cosmogenic models: & & & \\
$\quad$ Protheroe et al. 1996[11] & 0.6 & 13 & 44 \\
Engel et al. 2001[3] & 0.33 & 11 & 38 \\
$\quad$ Kotera et al. 2010[12] & 0.5 & 13 & 38 \\
Strong evolution models: & & & \\
$\quad$ Engel et al. 2001[3] & 1.0 & 34 & 120 \\
Kalashev et al. 2002[13] & 5.8 & 41 & 312 \\
Barger et al. 2006[14] & 3.5 & 32 & 91 \\
Yuksel et al. 2007[15] & 1.7 & 50 & 156 \\
Mixed-Iron-Composition: & & & \\
Ave et al. 2005[16] & 0.01 & 1.3 & 2.5 \\
Stanev 2008[17] & 0.0002 & 0.23 & 0.3 \\
Kotera et al. 2010[12] high & 0.08 & 2.4 & 6.4 \\
Kotera et al. 2010[12] low & 0.005 & 0.76 & 1.4 \\
Waxman-Bahcall (WB) fluxes: & & & \\
WB 1999, evolved[18] & 1.5 & 17 & 98 \\
$\quad$ WB 1999, standard[18] & 0.5 & 5.9 & 35 \\
IceCube PeV $E^{-2}$ power-law & & & \\
IceCube 2015 [19] & $\ldots$ & 2.9 & 6.10 \\
$\quad$ & & &
\end{tabular}


prove sensitivity to neutrino-induced radio-impulsive transients by a factor of $\gtrsim 100$ over ANITA.

These proceedings present an overview of the EVA mission concept and recent technology developments. The EVA team has performed extensive reflector antenna simulations using independent electromagnetics computational techniques for the surface of a SPB to validate the original estimates indicating a $\gtrsim 30 \mathrm{dBi}$ gain antenna is achievable. During September 2014, the EVA team performed tests on a $1 / 20$ th scale model SPB equipped with a reflector antenna on its surface to demonstrate deployment and provide validation of the electromagnetic antenna simulations.

\section{ExaVolt Antenna Mission Concept}

EVA will develop and deploy the largest-aperture radio telescope ever flown on a balloon payload, several thousand square meters, to be used to extend the radio-frequency collection power for neutrino impulses by a factor of 100 or more over any previous experiment. This will be accomplished using a portion of the physical balloon film itself as the radio reflector, with a toroidal geometry as indicated in Fig.2, where an approximately $10 \mathrm{~m}$ high equatorial section of the balloon surface is covered with RF reflective film. An incoming plane wave impulse enters the balloon below the lower reflector rim opposite the active focusing area that will apply for that direction. Once reaching the opposite side of the balloon reflector surface (a geometry that is locally an offaxis segment of a spheroidal mirror), the incoming plane wave impulse is focused onto a focal plane inside the balloon. Although only a portion of the area of the reflective region contributes to radio signal collection from any given direction, this area is still of order 100 square meters or more, equivalent to an $11 \mathrm{~m}$ diameter single-dish with uniform azimuth coverage, and a usable range of 10-15 degrees in elevation angle.

The proposed design centers on a novel use of a toroidal reflector as outlined above in Fig. 2. Radio reflector antennas based on a toroidal geometry were first described by Kelleher and Hibbs in 1953 [20], and Peeler and Archer in 1954 [21] and summary analyses may be found in several modern antenna textbooks [22]. Further details of our design process can be found in [9] and [23].

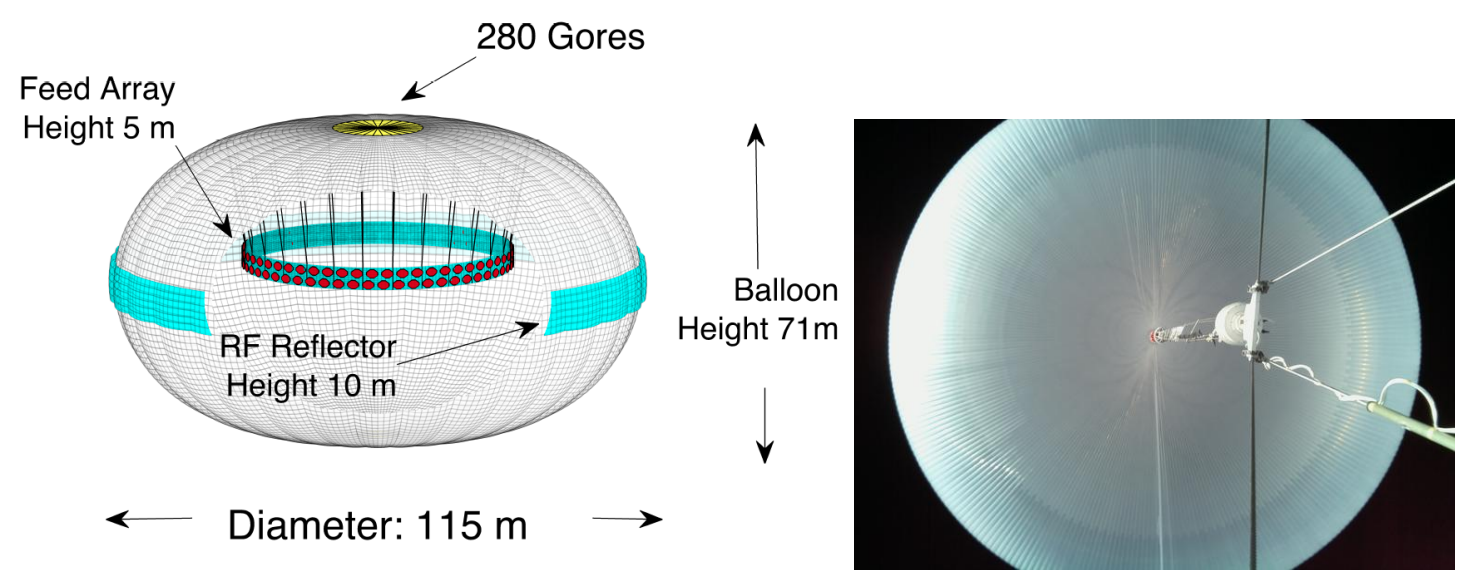

Figure 2: 3D schematic model of the EVA fullscale 18.7 Mcft super-pressure balloon. The reflector panels (light blue) are shown as a cutaway in the front portion, to reveal the internal feed array.

Figure 3: View of the 18.7 Mcft fully-inflated SPB at float during the 2014 flight. (courtesy of B. Stilwell, CSBF) 
At the focal plane, a set of feed antennas, which are flexible planar patch antennas affixed to the surface of an inner membrane of appropriate size to approximately match the focal plane angle, receive the focused plane wave. The polyethylene surface and load tendon material of the balloon play no role in any of the wave propagation, as these materials are completely transparent and extremely thin on the scale of the wavelengths of interest here.

\section{Reflector Antenna Modeling}

The EVA team has created detailed antenna models for the super pressure balloon (SPB) geometry involving the currently available $18.7 \mathrm{Mcft}$ balloon with approximately $57 \mathrm{~m}$ radius, and this model is the basis for most of the results provided here. The EVA antenna models now use three independent antenna modeling software suites: The method of moments based Numerical Electromagnetic Code v.4 (NEC4) [24], the physical optics based General Reflector Antenna Simulation Program (GRASP) [25], and the finite difference in the time domain based XFdtd v.7 [26]. A photograph of an actual fully inflated 18.7 Mcft SPB used in the 2014 Antarctic NASA LDB campaign is shown in Fig. 3.

PB was used also to create the 1/20th scale EVA balloon design as specified by the EVA team. The simulated balloon is based on the Euler-elastica, which is the analytically derived curve for the SPB surface [23]. It assumes 280 total gores, consistent with the $18.7 \mathrm{Mcft}$ balloon. The model assumes a case where the fully-inflated lobe center is about $10 \mathrm{~cm}$ further out than a circular arc joining the tendons. This value is about $20 \%$ of the wavelength at $600 \mathrm{MHz}$ which is currently the upper end of our design frequency.

The feed array system for EVA occupies the surface of an inner $5 \mathrm{~m}$-high cylindrical band which is deployed gradually during the ascent of the balloon. Once the balloon is fully inflated, this element comes into slight tension, causing the feed array to achieve its final deployed form.

The model NEC4 analysis was done by iterative optimization of the gain vs. frequency and angle for different locations of a probe antenna which acted as the feed excitation for the larger toroidal section. Starting values for the best feed location were based on the sagittal and transverse foci of the astigmatic reflector. We found that the best gain values and apparent circle of least confusion for the focused antenna beam tended to favor that equatorial radius of curvature, primarily because there was more reflective area available at the larger focal distance, and the depth of focus was also larger. We did not adjust the width of the vertical reflective section; this is another parameter that could be

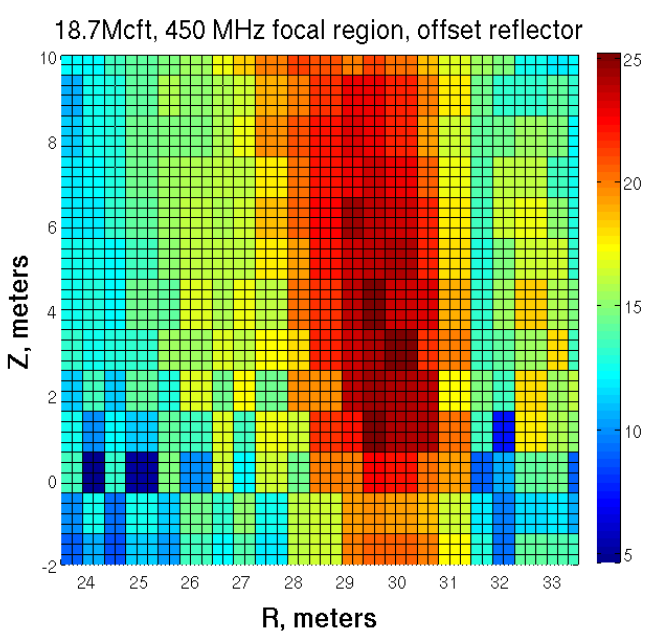

Figure 4: Estimated directivity vs. position in the $R-z$ plane in the focal region of the $18.7 \mathrm{Mcft}$ balloon, as given by our NEC4 model. optimized. Our NEC model filled the aperture of the reflector with the equivalent of $10 \mathrm{~cm}$ diameter "wires" at a spacing of about $35 \mathrm{~cm}$, corresponding naively to about $30 \%$ reflectivity of the surface; 
this value was chosen as the closest that we could place the elements while still maintaining robust numerical stability for the calculation. Thus the estimated antenna gains can be expected to show some improvement with fully-filled reflective sections. Fig. 4 shows one of the primary results of the simulation: a section view through the region of best focus, in this case in the $R-z$ plane, where $R$ is the cylindrical radial coordinate, and $z$ the axial coordinate vertically through the balloon center. The center of the reflective band is offset $2.5 \mathrm{~m}$ above the equator in this simulation to give the best possible down-looking field-of-view without occultation. This then yields a focal region, seen in red, with a radial depth of focus of order \pm 1 meter thick, and a nearly vertical extent of the focal plane of order 6 meters FWHM. The peak gains observed in this simulation are of order $25 \mathrm{dBi}$ at the focus, but are likely underestimates of the true gain due to the sparse wire-grid sampling.

The simulation with XF7 is quite distinct from the other modeling in that it takes place in the time domain without the direct use of physical optics or methods of moments. Instead FDTD methods use a gridded space and enforce classical electrodynamics requirements (eg. Maxwell's equations) at all times and locations in the space, resulting in a complete simulation of the evolution of radiation, surface currents, near and far-field behavior, and transient responses. For this simulation we used a high-fidelity model of the EVA reflector for the full $18.7 \mathrm{Mcft}$ balloon, and stimulated it with a radio impulse plane wave, very similar to the anticipated ultra-high energy particle signal. Results are shown in Fig. 5, where each pane from left to right shows various stages in the reflection and focusing of the incident plane wave impulse. In each frame the reflector is seen as a semicircular arc facing right, and the pulse energy is reflected and focused to a small region at a distance of about $R=29-30$ meters from the center in the final frame, consistent with the NEC and GRASP results. The final frame in Fig. 5 shows the pulse amplification, normalized to give directivity gain, as a function of light travel distance in the system. The gain is about $24 \mathrm{dBi}$.
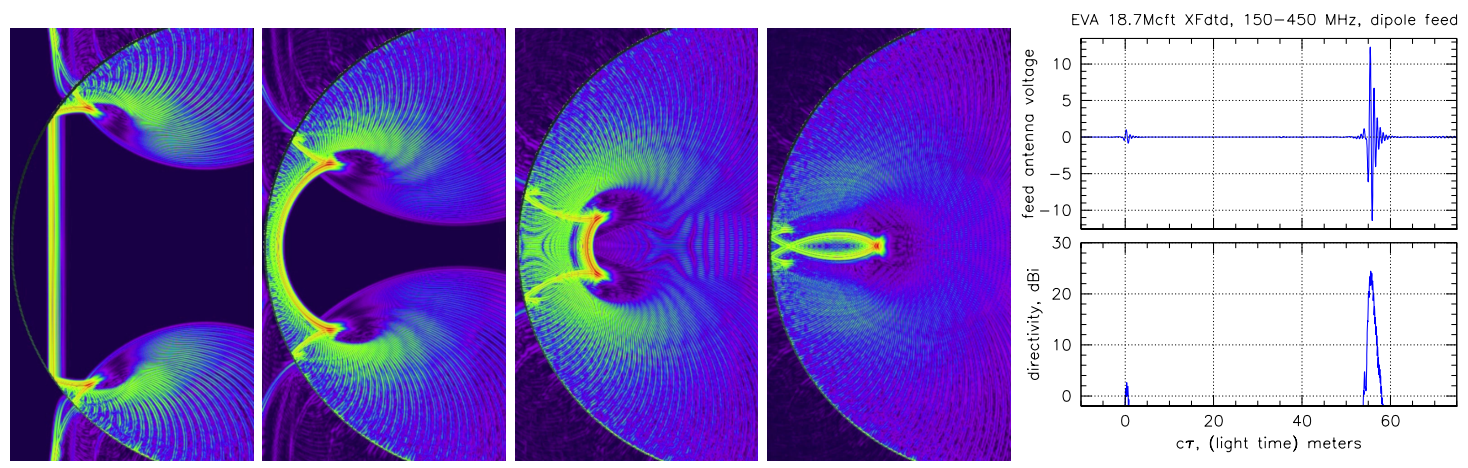

Figure 5: XFdtd results for EVA 18.7 Mcft balloon: Left panels, evolution of plane wave impulse (incident from the right side, viewed from above) as it is focused by the EVA reflector; the time sequence moves from left to right. Right: time series of the arriving pulse, converted to directivity gain of the system, and peaking at about $24 \mathrm{dBi}$.

GRASP is a simulation tool primarily aimed at the design of large radio dishes and powered reflectors, but it can be used with a numerically specified reflector surface, such as the design for the 18.7 Mcft balloon. Once the surface is defined, GRASP then requires specification of the feed, and in our studies with GRASP, we have identified important features in the gain which depend on the choice of feed geometry. Thus GRASP was able to reproduce gain results as estimated by both NEC4 and XF7, using low gain feeds such as dipoles, with the EVA reflector giving typical gains of order $23-27 \mathrm{dBi}$ in the UHF frequency band. In addition, by using feeds that 
were specifically designed to match the receiving area of the large reflector, GRASP has indicated directions for significant improvement in the overall gain of the system, up to the range of 32 dBi. Fig. 6 summarizes the GRASP results, showing beam pattern data which complements that indicated in Fig. 4 above, where the effective depth-of-focus was probed by NEC4.

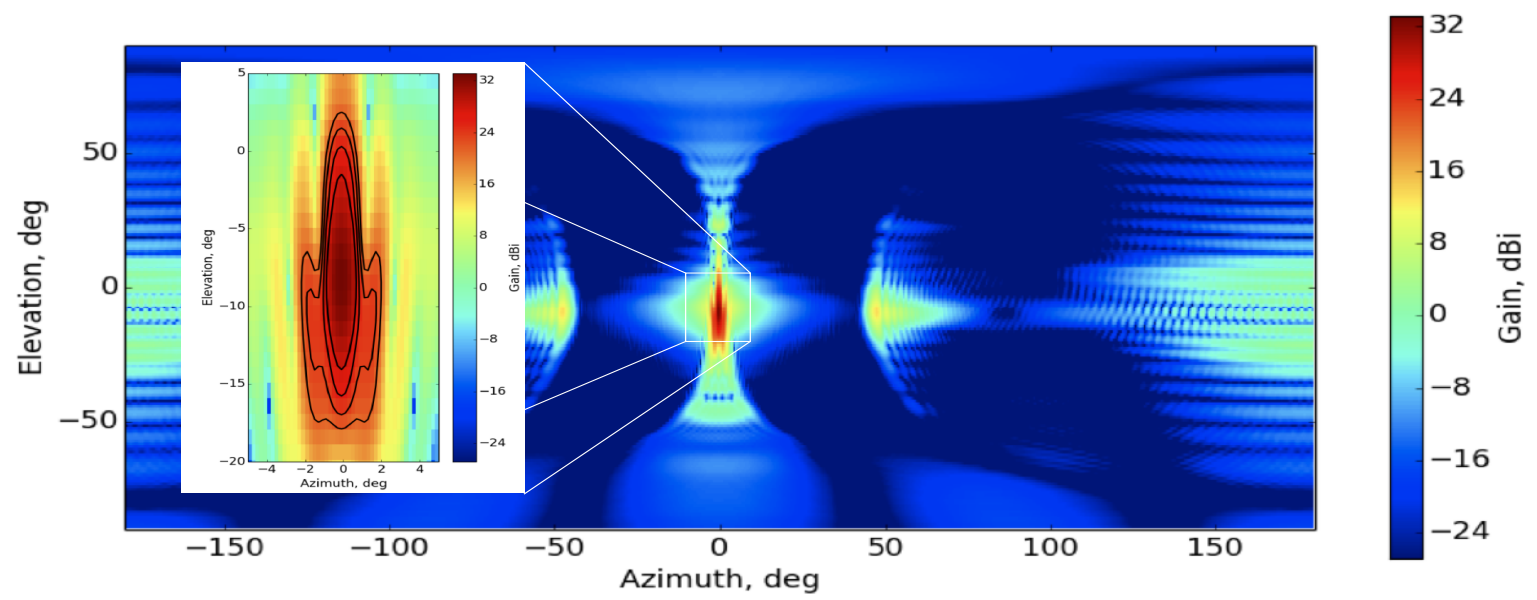

Figure 6: GRASP model power pattern for 18.7 Mcft EVA model, using a feed with response matched to the reflector.

\section{The 1/20th-scale Balloon Test}

In September 2014, the EVA collaboration performed a hangar test, at NASA Wallops Flight Facility, of a $5.7 \mathrm{~m}$ diameter SPB constructed to our specifications by Aerostar International, the vendor responsible for all current NASA flight balloons. This balloon included 50-cm high aluminized-mylar reflective panels married to the balloon at the equator by the vendor using polyethylene adhesive tape; these panels were used in lieu of the silvered polyethylene film we plan for the full-scale article, as the latter film is still under development. The microwave reflectivity of the panel material was measured using a waveguide reflectometer and found to be excellent. A view of the fully-deployed balloon during the test is shown in Fig. 7.
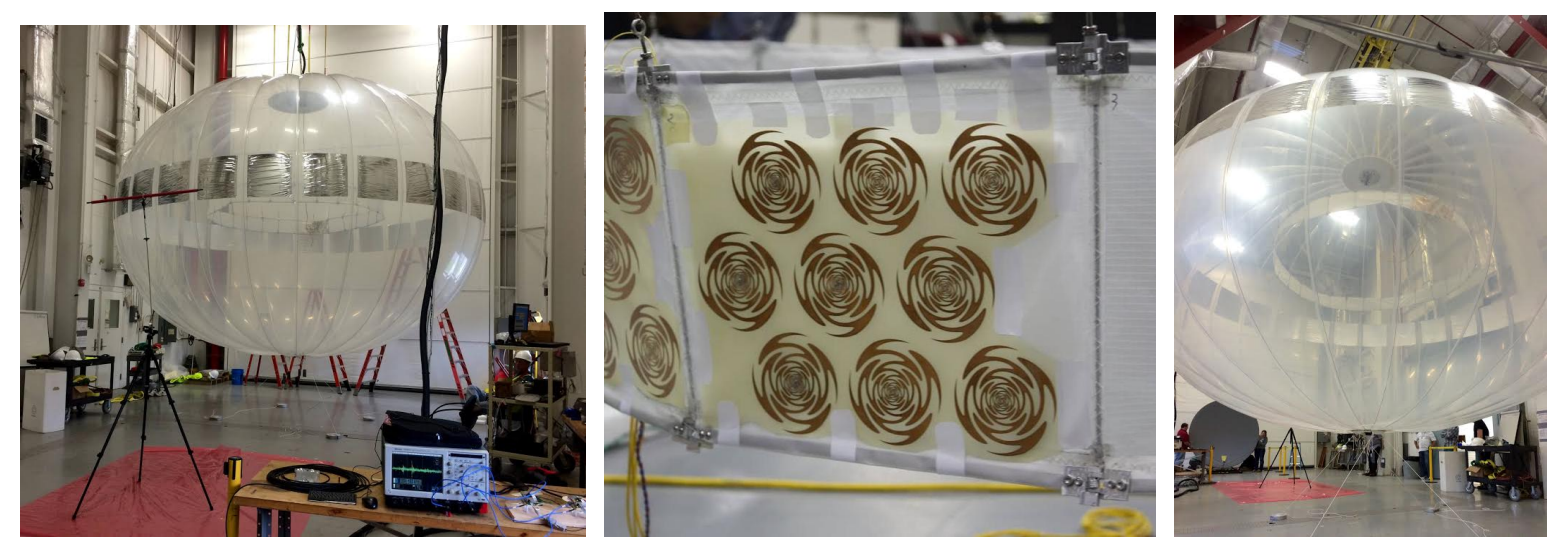

Figure 7: Left: Fully deployed $20 \mathrm{ft}$ diameter balloon, and DAQ system in foreground. Center: detail of sinuous patch antenna array. Right: view of fully deployed internal feed array, from below the balloon.

Prior to inflation of the balloon, we inserted a scale-model of the antenna feed array membrane through the top end-plate of the balloon, which was flanged with a $1 \mathrm{~m}$ opening. The feed array 
was folded to accommodate insertion, and was married to internal support lines which drew it out of its folded position during inflation, and eventually provided both support and a slight outward tension to give a fully deployed feed array. The feed array membrane was instrumented with dual polarization sinuous patch antennas over a portion of its circumference, and four of these antennas were also instrumented with microwave receivers coupled to RF-over-fiber transceivers which inserted the RF signals into optical fiber, as shown in Fig. 7. The fibers passed through the top end-plate bulkhead, and then to an external receiver, which allowed us to capture the received signals at the balloon focal plane with minimal transmission losses.

Our goals in this test were to demonstrate that it was possible to deploy a feed membrane antenna array within a superpressure balloon, and that the optics of the balloon and patch antennas would function as predicted by antenna models when subject to an external impulsive plane-wave stimulus. To create this stimulus, a 2.6 meter offset parabolic dish was used as an RF collimator, with a broadband dual-ridge horn at its feed position giving microwave impulses. The impulse used was short enough that it could be observed both as it passed by the feed patch antenna initially, and then as it returned again after being focused by the reflective panels. We made several compromises on the scalability for the sake of the test: The number of gores was set at 28 for this balloon, rather than 280 , to keep construction simple; and the width of the colli-

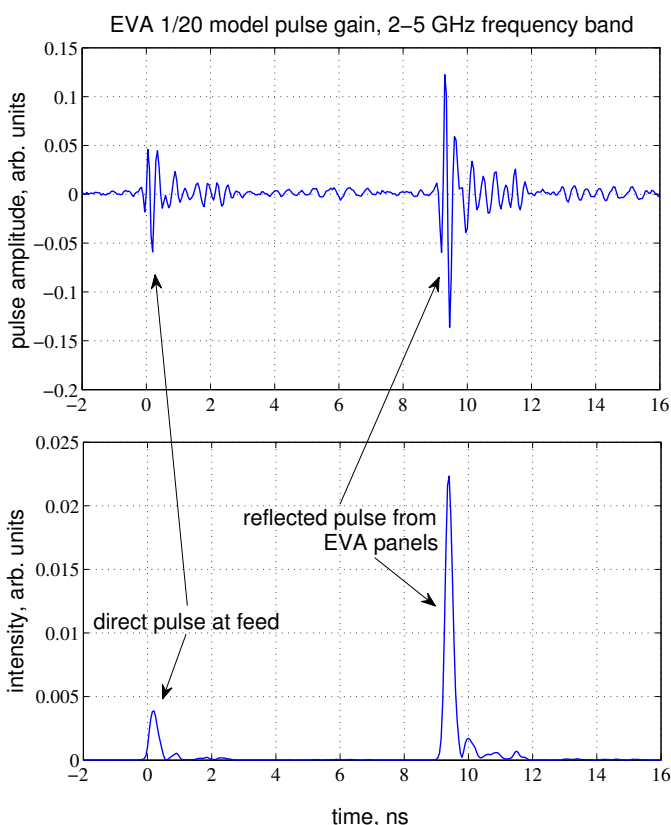

Figure 8: Direct and focused pulse as measured in our 1/20th scale EVA test, both received voltage (top) and intensity (bottom). mation dish was much smaller than the receiving width of the reflective panels due to constraints of portability of the dish. These compromises were understood and incorporated in the models. The results of the microwave test are summarized in Fig. 8. In the upper pane, the received amplitudes are seen, both for the low-gain feed, and after focusing by the reflective panels; the bottom pane shows the corresponding intensity of the signals. It is evident that the temporal coherence of the arriving impulse is preserved in the focused pulse. The observed pulse gain also matches our model estimates within about $2 \mathrm{~dB}$, a solid validation of the simulation tools and our methodology. We have thus improved the credibility of the estimates for the full-scale article.

Acknowledgements The EVA collaboration is grateful to NASA, the U.S. National Science Foundation, the U.S. Department of Energy, and the NASA Wallops Flight Facility. Part of this research was carried out at the Jet Propulsion Laboratory, California Institute of Technology, under a contract with the National Aeronautics and Space Administration. Government sponsorship acknowledged. 


\section{References}

[1] V. S. Berezinsky and G. T. Zatsepin, Phys. Lett. B 28, 423 (1969).

[2] C.T. Hill and D, N. Schramm, Phys. Rev. D 31,564 (1985); F.W. Stecker, Phys. Rev. Lett. 21, 1016-1018 (1968).; D. Seckel and T. Stanev, Phys. Rev. Lett. 95141101 (2005).

[3] R. Engel, D. Seckel, \& T. Stanev, 2001, Bartol Research Institute Report Number BA-01-01 (astro-ph/0101216) and references therein.

[4] J. Linsley, Phys. Rev. Lett. 10, 146 (1963); see also Proc. of the Int'l Cosmic Ray conference, Jaipur 1963, ed. R. Daniel et al., (Commercial Press, Bombay), vol. IV, 77.; D. J. Bird et al., Phys. Rev. Lett. 71, 3401 (1993); H. Hayashida et al., Ap. J. 522, 225 (1999); C. C. H. Jui et al., 26th International Cosmic Ray Conference Invited, Rapporteur, and Highlight papers (AIP Conf. Proc 516), 370 (2000); T.Abu-Zayyad, at. al., Astrophys. J. 557, 686 (2001); M. Takeda et al., Astroparticle Physics 19, 447-462 (2003); Schuessler, F. [Auger Collaboration], Phys. Lett. B, 2009, in press. (arXiv:1002.1975); A. Aab et al. (Pierre Auger Collaboration) Phys. Rev. D 90, 122005, Published 31 December 2014; see also http: / / arxiv.org/abs/1409.5083

[5] I. Kravchenko et al. , Phys. Rev. D 85, 062004 (2012)

[6] P. Allison, et al. [ARA Collaboration], Astroparticle Physics, Volume 35, Issue 7, February 2012, 457.

[7] S. W. Barwick, et al. , arXiv:1410.7369, submitted to Astroparticle Physics, 2015

[8] P. Gorham et al. [the ANITA collaboration], Phys. Rev. D82, 022004 (2010).

[9] P. W. Gorham et al. , Astroparticle Physics, 35, 242, (2011).

[10] A. Connolly, for the ARA collaboration.

[11] R. J. Protheroe \& P. A. Johnson, Astropart. Phys. 4 (1996) 253.

[12] Kumiko Kotera, Denis Allard, Angela V. Olinto, JCAP 1010:013,2010; arXiv:1009.1382.

[13] O. E. Kalashev, et al.,Phys. Rev. D66, 063004 (2002).

[14] V. Barger, P. Huber, D. Marfatia, Phys. Lett. B 642, 333 (2006).

[15] H. Yuksel, M. D. Kistler, Phys. Rev. D 75, 083004 (2007); astro-ph/0610481.

[16] M. Ave, N. Busca, A. V. Olinto, A. A. Watson, and T. Yamamoto, Astropart. Phys. 23 (2005) 19.

[17] T. Stanev, Nucl. Instrum. Meth. A588: 215-220, (2008).

[18] E. Waxman and J. N. Bahcall, Phys. Rev. D 59, 023002 (1999); J. N. Bahcall and E.Waxman, Phys. Rev. D 64, 023002 (2001).

[19] IceCube Collaboration: M. G. Aartsen et al. , Flavor Ratio of Astrophysical Neutrinos above 35 TeV in IceCube, submitted to PRL, 2015, http://arxiv.org/abs/1502.03376.

[20] K. S. Kelleher, and H. H. Hibbs, Naval Research Laboratory Report No. 4141, May 1953.

[21] G. D. M. Peeler, and D. H. Archer, IRE Convention Record, 1954, 242-247.

[22] R. C. Johnson and H. Jasik, Antenna Engineering Handbook, (McGraw-Hill: New York), 2nd edition, 1984; E. A. Wolff, Antenna Analyis, (Wiley and Sons: New York), first edition, 1966.

[23] F. Baginski and P. Gorham, 2013 IEEE Aerospace Conference, 2013, March 2013, pp.1-8.

[24] G. J. Burke, E. K. Miller, and A. J. Poggio, IEEE Int'l Symp. National Radio Science (URSI), 2004.

[25] http://www.ticra.com/products/software/grasp

[26] http://www.remcom.com/xf7 